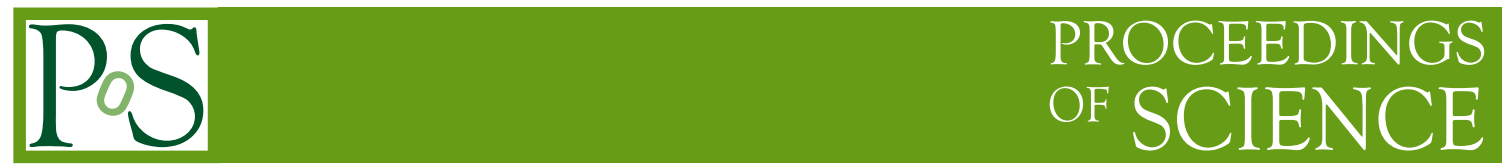

\title{
Antideuteron sensitivity studies at LHCb
}

\author{
Sophie Baker*† \\ Imperial College London \\ E-mail: sophie.katherine.baker@cern.ch
}

Preliminary studies into the potential measurement of the (anti)deuteron production cross-section in proton-proton collisions at $\mathrm{LHCb}$ are presented. The expected (anti)deuteron yield is seen to be low relative to other charged tracks, but the excellent particle identification capabilities of $\mathrm{LHCb}$ may ensure that a measurement is possible.

Sixth Annual Conference on Large Hadron Collider Physics (LHCP2018)

4-9 June 2018

Bologna, Italy

* Speaker.

${ }^{\dagger}$ On behalf of the LHCb Collaboration 


\section{Introduction}

Measurements of antideuterons at HEP experiments can help to constrain systematic uncertainties in indirect dark matter searches. These searches look for excess signals of antimatter in cosmic rays; no conclusive evidence for dark matter has been seen using antiproton or positron signals, but heavier antimatter, such as antideuterons, has lower backgrounds compared to these [1]. Uncertainties in the knowledge of the propagation of charged particles through the universe, as well as the production of secondary antideuterons by cosmic ray spallation, currently limit the power of these antideuteron searches.

Neither deuterons nor antideuterons have been measured at the LHCb experiment, but due to the detector's excellent particle identification capabilities, it may be possible. A thorough investigation into this possibility is presented. A production cross-section measurement would contribute to the indirect dark matter field, as well as having implications for similar measurements at $\mathrm{LHCb}$ in the future.

\section{Production models}

There are two popular models for the production of deuterons ${ }^{1}$ from pairs of high energy nucleons. The coalescence model [2] dates back to 1963, and has been used widely and successfully since then. It proposes that protons and neutrons bind when they are close enough in momentum space. The model takes the form of a step function in the centre of mass momentum difference of the nucleon pair, shown in dashed blue in Fig. 1, where the threshold momentum difference for

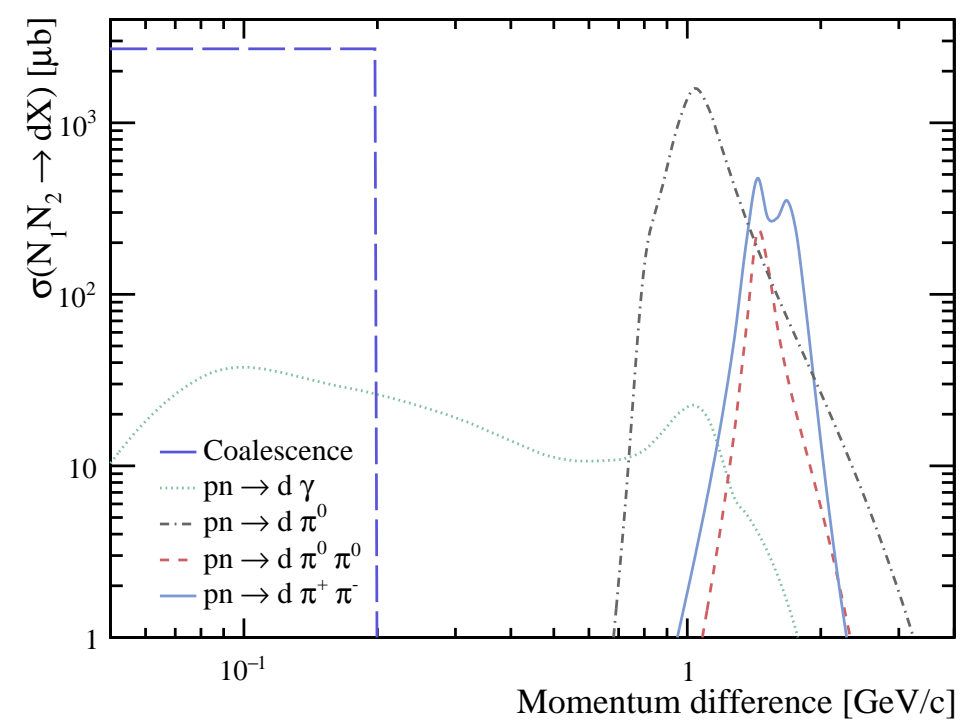

Figure 1: Cross-sections for deuteron production channels for the coalescence [2] and cross-section [3] models as functions of the momentum difference of binding proton-neutron pairs in their centre of mass frame.

\footnotetext{
${ }^{1}$ Charge conjugates are implied for the remainder of these proceedings.
} 


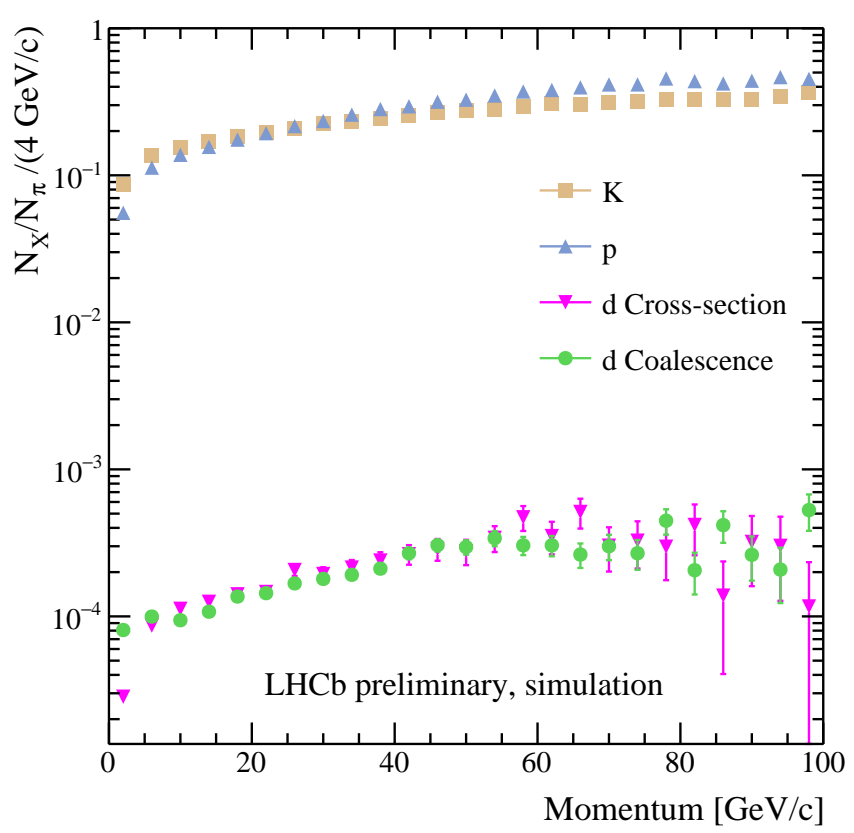

Figure 2: Expected deuteron yield relative to pion yield in bins of momentum, from simulation of the coalescence and cross-section models in $p p$ collisions in the LHCb acceptance. Relative proton and kaon yields are also shown.

binding is taken from measurement. The cross-section model [3] was put forward more recently, and includes mechanisms for $p p, p n$ and $n n$ pairs to bind through multiple channels; some examples of $p n$-binding cross-sections as functions of centre of mass momentum difference are shown in Fig. 1. This model uses a probabilistic approach to determine if a binding is successful, with the normalisation taken from measurement.

\section{Simulation}

The two deuteron production models have each been appended to existing LHCb simulation in order to estimate the expected yield of deuterons in $p p$ collisions at the LHC. For each simulated collision, pairs of protons and neutrons are compared. Their momentum difference is computed, and the chosen model is used to evaluate the likelihood that they will bind. If successful, a vertex is added to the simulated event, with an outgoing deuteron. The deuteron yield resulting from these two models is shown in Fig. 2 as a function of pion yield in bins of momentum within the LHCb acceptance. For both models, the deuteron yield is approximately $10^{-4}$ times the pion yield. The relative yields of protons and kaons are also shown. 


\section{Charged particle identification at LHCb}

The LHCb detector was designed to have excellent charged particle identification, in order to differentiate between $B$-hadron decays. Ring imaging Cherenkov (RICH) detectors and tracking stations are used to differentiate between protons, kaons, pions, muons and electrons $[4,5]$. Deuterons can be identified in the same way.

The momentum of each charged track is measured in the tracking stations upstream and downstream of the RICH detectors. Cherenkov photons within a small angle with respect to each track are then isolated, and the photon distributions are compared to hypothetical distributions for different charged particles with the same momentum as the measured track. The RICH algorithm outputs the difference in log likelihood for each track to originate from each of the charged particle hypotheses compared to the pion hypothesis. Selections can be applied to these log likelihoods to choose different particle types.

\section{Selection efficiency}

In order to know the true yields of particles, the efficiency for selecting a given track type using the log likelihoods from the RICH must be known. Data samples of tracks of known particle type are selected in order to determine efficiencies accurately [6]. These samples are selected kinematically, without use of particle identification variables. Selections based on the log likelihoods are then applied to the known particle tracks, and the selection efficiency is the number of tracks surviving the selection, divided by the total number of tracks in the sample [5]. There are no high purity data samples for deuterons, so their efficiencies are taken from simulation.

Given the low expected deuteron yield, it is important that other charged particles are not misidentified as deuterons, as this would dominate the signal. In order to reject background from protons, kaons and pions, a combination of cuts is applied, to disfavour all three. In Fig. 3, the

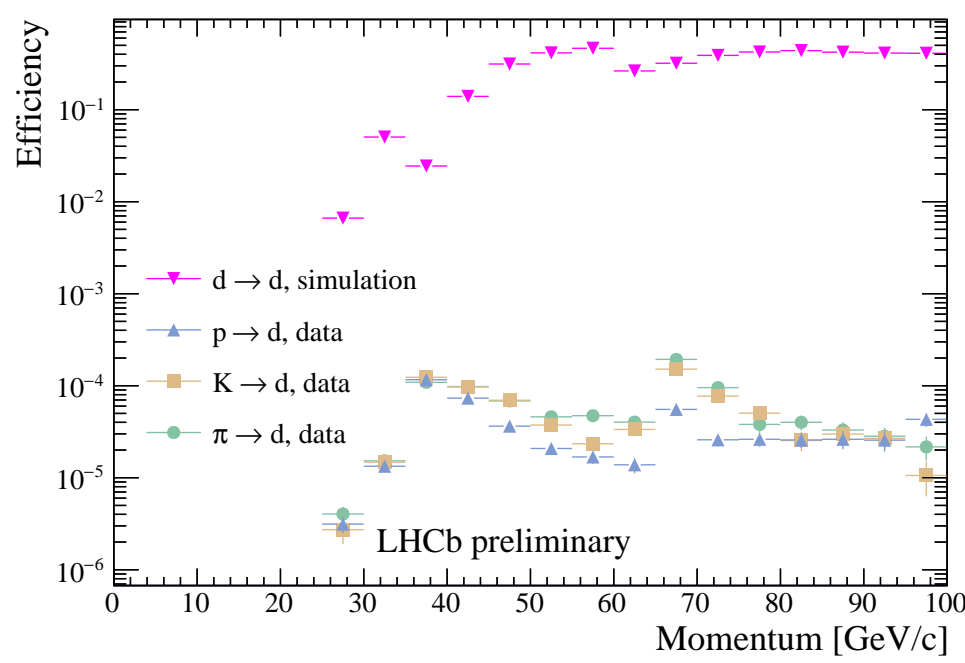

Figure 3: Deuteron selection efficiencies for deuterons from simulation, and protons, kaons and pions from high purity data samples. 
efficiencies for identifying proton, kaon, pion and deuteron tracks as deuterons are shown in bins of momentum for tight selections, taken from simulation for the deuteron tracks, and from the high purity data samples for the other track types. Large variations in the efficiencies occur at the momentum thresholds for Cherenkov photon emission in each of the RICH detectors. At these momenta, the power to discriminate between particle species changes, before leveling off further away from the thresholds. For this tight selection, the efficiency for selecting a pion as a deuteron is reduced to the level of $10^{-4}$, whilst maintaining a high efficiency of deuteron selection; this brings the expected signal and background to similar levels.

\section{Measurement potential}

With the high statistics data collected at the LHCb experiment in 2016 and 2017, and the efficiencies for deuteron selection shown above, it is expected that a measurement of the crosssection of prompt deuteron production from $p p$ collisons at a centre of mass energy $\sqrt{s}=13 \mathrm{TeV}$ will be possible. This measurement will open the door to further deuteron measurements at LHCb, and will contribute to reducing the uncertainties involved in indirect dark matter searches.

Alternative particle identification variables could also be used for this measurement, such as those taken from neural networks, trained to differentiate between particle types. Combining identification techniques will increase the sensitivity and further reduce the backgrounds.

\section{References}

[1] T. Aramaki et al., Review of the theoretical and experimental status of dark matter identification with cosmic-ray antideuterons, Phys. Rept. 618 (2016) 1, arXiv:1505.07785.

[2] J. I. Kapusta, Mechanisms for deuteron production in relativistic nuclear collisions, Phys. Rev. C21 (1980) 1301.

[3] L. A. Dal and A. R. Raklev, Alternative formation model for antideuterons from dark matter, Phys. Rev. D91 (2015) 123536, arXiv: 1504.07242.

[4] M. Adinolfi et al., Performance of the LHCb RICH detector at the LHC, Eur. Phys. J. C73 (2013) 2431, arXiv:1211.6759.

[5] L. Anderlini et al., The PIDCalib package, LHCb-PUB-2016-021.

[6] R. Aaij et al., Selection and processing of calibration samples to measure the particle identification performance of the LHCb experiment in Run 2, arXiv:1803.00824. 